\title{
Quality assessment for real out-of-focus blurred images
}

\author{
Yutao Liu ${ }^{\mathrm{a}, *}, \mathrm{Ke} \mathrm{Gu}^{\mathrm{b}}$, Guangtao Zhai ${ }^{\mathrm{c}}$, Xianming Liu ${ }^{\mathrm{a}}$, Debin Zhao ${ }^{\mathrm{a}}$, \\ Wen $\mathrm{Gao}^{\mathrm{a}, \mathrm{d}}$ \\ ${ }^{a}$ School of Computer Science and Technology, Harbin Institute of Technology, Harbin \\ 150001, China \\ ${ }^{b}$ School of Computer Engineering, University of Technology, Sydney, Australia \\ ${ }^{c}$ Institute of Image Communication and Information Processing, Shanghai Jiao Tong \\ University, Shanghai 200240, China \\ ${ }^{d}$ National Engineering Laboratory for Video Technology, Key Laboratory of Machine \\ Perception, School of Electrical Engineering and Computer Science, Peking University, \\ Beijing 100871, China
}

\begin{abstract}
Images are vulnerable to different kinds of distortions, such as blur, noise, blockiness etc, which all degrade the image quality. Among the distorted images, out-of-focus blurred images are frequently-encountered and occupy a large proportion. However, few efforts have been done to quality evaluation for these images. In this paper, we devise a dedicated quality evaluation scheme to automatically infer the quality of out-of-focus blurred images, which is named GPSQ (Gradient magnitude and Phase congruency-based and Saliency-guided Quality model). In GPSQ, a pair of low-level features, including gradient magnitude (GM) and phase congruency (PC), are extracted to characterize the image local blurriness. Then saliency detection is performed on the image to generate a corresponding saliency map. Finally, we weight the local structure map with the saliency map to estimate the visual quality of the out-of-focus blurred image. Experimental results demonstrate the proposed GPSQ delivers high consistency with subjective evaluation results.
\end{abstract}

Keywords: Visual quality assessment, out-of-focus blur, blind/no-reference, phase congruency, visual saliency

\footnotetext{
* Corresponding author

Email address: liuyutao2008@gmail.com (Yutao Liu)
}

Preprint submitted to Journal of $E^{A} T_{E} X$ Templates

January 27, 2017

(C) 2017. This manuscript version is made available under the Elsevier user license http://www.elsevier.com/open-access/userlicense/1.0/ 


\section{Introduction}

The popularization of hand-held cameras makes the acquisition of images much convenient nowadays. However, due to inappropriate camera settings or photographing in a casual manner, the images thus acquired often suffer from 5 a variety of distortions, such as blur, noise, contrast distortion etc., which all degrade the images' quality. Among the distorted images, images with out-offocus blur are frequently-encountered and occupy a large proportion. Hence, in this work, we put forward an objective quality model dedicated to measuring the quality of the out-of-focus blurred images. The objective quality assessment approach can be applied to monitoring the image quality during acquisition or cull the unacceptable images with out-of-focus blur.

In general, existing image quality assessment (IQA) approaches can be classified into three classes, namely no reference (NR), reduced reference (RR) and full reference (FR) metrics. The two most representative FR models are prob15 ably the peak signal-to-noise ratio (PSNR) and the structural similarity index (SSIM) 1]. PSNR measures the image quality through the energy of the residual of a distorted image against its associated reference one. While SSIM infers the visual quality by comparing the similarity in structures. In recent years, to better model the HVS in quality assessment, researchers have employed high level characteristics of the HVS into the objective algorithm designment, such as visual saliency. Representative works can be referred to in 2, 3] 4, 5, where [2] 3 ] introduced saliency into IQA for algorithm establishment while [4 [5] analyzed important issues about the application of saliency in IQA tasks.

The second category of IQA methods is the RR IQA methods based on partial information or some representative features from the reference image for quality estimation. The authors in [6 proposed an information theoretic RR IQA algorithm that measures the mean deviation between reference and projected distorted images from the viewpoint of scaled entropies of wavelet coefficients. In [7, the authors designed a free-energy based distortion metric so (FEDM) based on the recent discovery of free-energy theory in neuroscience. In 
[8], the authors constructed IQA model by combining the merits of the human visual system (HVS), such as contrast sensitivity function, multi-scale geometric analysis, and the Weber's law of just noticeable difference.

However, in most cases the reference image is absent, then FR and RR methods become both invalid. In this regard, NR IQA provides the only way to measure the image quality. The first type of NR IQA approaches is general-purpose, which is designed to handle images without knowing the distortion type in advance. These methods are usually developed in two steps, feature extraction followed by training a prediction module for quality evaluation. Representative general-purpose NR methods contain DIIVINE 9, BLIINDS-II [10, BRISQUE [1] and NFERM [12] etc. The second type of NR methods is proposed to deal with images with specific distortion, e.g., JPEG [13] 14, blur [15, noise [16] or contrast change [17] 18] etc.

In this paper, our attention is focused on the quality assessment of a special 45 kind of distorted images, namely out-of-focus blurred images. As we mentioned before, these images are frequently-encountered in reality while specific quality evaluation methods for these images are still limited. Certainly, the out-of-focus blurred images can be assessed by the general-purpose NR methods due to their general QA ability for distorted images. However, compared with the generalpurpose methods, the specific blurriness assessment methods are more pertinent to tackle this kind of images. In literature, early attempts for blurriness estimation concentrated on the image edges. For instance, the authors in [15] proposed the blur metric based on the pair edge detectors in vertical and horizontal orientations. Just noticeable blur (JNB) was proposed by measuring the blur around the edges. In addition, image blur can also be characterized by its spectral behaviors. An efficient wavelet-based local and global image sharpness assessment method (FISH) [19] was developed by calculating the Log-energy of the coefficients in the DWT domain. In [20, the spectral and spatial sharpness metric (S3) works in combining the measurements of the total spatial variation and the 60 slope of the magnitude spectrum. Although general-purpose NR methods and blurriness assessment methods both have the capacity of assessing the out-of- 
focus blurred images, these methods may become ineffective or insufficient as the real out-of-focus blur exhibits complexity and irregularity over the image. The complexity and irregularity mainly lie in that the position of the out-offocus blur is unpredictable and its intensity is also spatially variant, which gives rise to challenges to existing IQA methods for accurately predicting the quality of out-of-focus blurred images. To tackle this, we devise a quality assessment scheme dedicated to the out-of-focus blurred images, which is named GPSQ (Gradient magnitude and Phase congruency-based and Saliency-guided Quality model). Since multi-domain features of the image can play complementary roles in quality assessment 21] 22] 23], we extract two low-level features GM and PC from the spatial and spectral domains respectively and then combine them to comprehensively characterize the image blurriness. Next we perform saliency detection on the out-of-focus blurred image and get a corresponding saliency map. Finally, we weight the localized structure map with the saliency map to derive a single score which denotes the visual quality of the out-of-focus blurred image. Considering chrominance information can also affect human perception of the image quality, we further extend GPSQ to GPSQ $c$ by incorporating the GMs of the image chromatic components. Through extensive experiments, we verify GPSQ/GPSQ ${ }_{c}$ works in high consistency with subjective opinions on the image quality.

The remainder of this paper is arranged as follows: Section 2 illustrates the proposed scheme GPSQ in detail. Experimental results and analyses are given in Section 3 . At last, we make conclusions of this paper in Section 4

\section{Methodology}

\subsection{GM extraction}

The HVS is largely adapted to extracting the structural information from the input image scenes as the structures in the image convey much critical information for the HVS to explain the input visual signals [1. Unfortunately, the introduction of out-of-focus blur leads to structure degradation, which hampers 
the HVS from interpreting the image normally and thus degrades the image visual quality. To effectively capture the image local structures, we calculate GM of the image, which can extract the image structures from the spatial domain [21]. Specifically, we firstly compute the image gradients by convolving the image $I$ with the Prewitt operator along two orthogonal directions as:

$$
\begin{gathered}
G_{x}(I)=\frac{1}{3}\left[\begin{array}{ccc}
1 & 0 & -1 \\
1 & 0 & -1 \\
1 & 0 & -1
\end{array}\right] \otimes I \\
G_{y}(I)=\frac{1}{3}\left[\begin{array}{ccc}
1 & 1 & 1 \\
0 & 0 & 0 \\
-1 & -1 & -1
\end{array}\right] \otimes I
\end{gathered}
$$

where $G_{y}(I)$ and $G_{x}(I)$ respectively stand for the image gradients along the vertical and horizontal directions, " $\otimes$ " denotes the convolution operator. Then GM of $I$ can be calculated as:

$$
G M=\sqrt{G_{x}^{2}+G_{y}^{2}}
$$

GM than the slight blur by comparing (e) and (f). This observation can also be verified according to the GM distributions shown in (g). Namely, the GM 


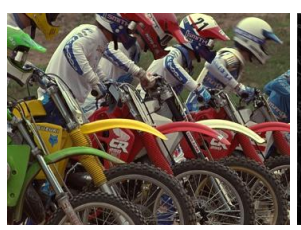

(a)

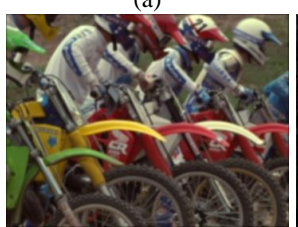

(b)

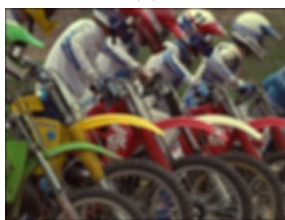

(c)

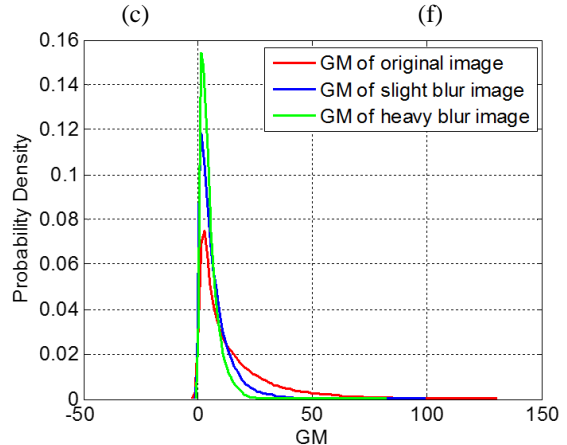

(g)

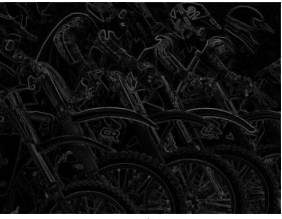

(d)

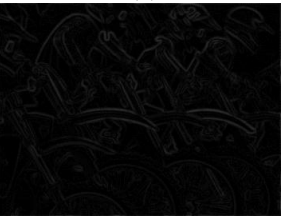

(e)

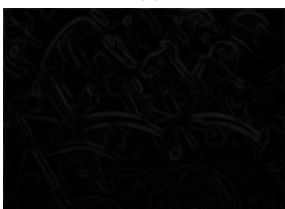

(f)

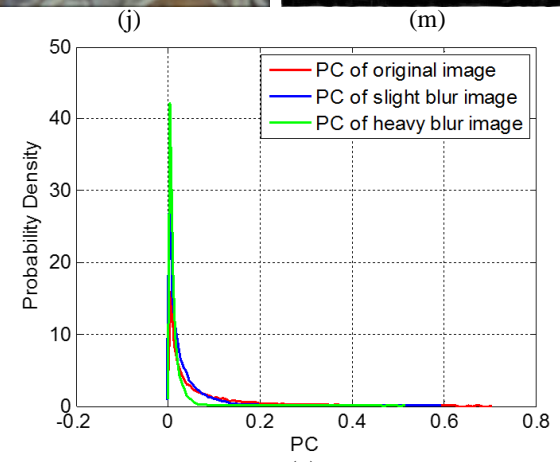

(n)

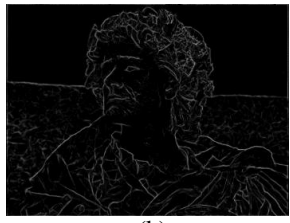

(k)

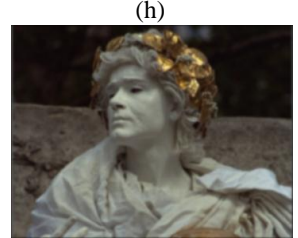

(i)

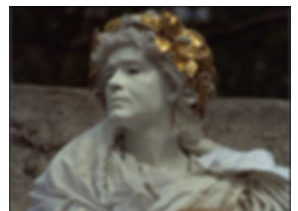

Figure 1: The example GM and PC images and their distributions: (a)(b)(c) are the images with no blur, slight blur and heavy blur respectively. (d)(e)(f) are the corresponding extracted GM images of (a)(b)(c). (g) gives the GM distribution. Likewise, (h)(i)(j) are the images with no blur, slight blur and heavy blur respectively. $(\mathrm{k})(\mathrm{l})(\mathrm{m})$ are the corresponding extracted PC images of $(\mathrm{h})(\mathrm{i})(\mathrm{j})$. (n) shows the PC distribution. 
distributions of the blurred images in blue and green lines are all deviated from that of the original image in red line, while the distribution of the slight blur image is closer to that of the original image than the distribution of the heavy blur image. Therefore, GM can characterize the image blurriness effectively.

\section{2. $P C$ extraction}

Besides extracting the image structures in the spatial domain with GM, we also consider the activities of structures in the frequency domain of the image. In the light of PC theory, the structure features can be perceived at those points where the Fourier components take maximum values in phase [24] 25] [26]. Therefore, the PC model offers us another perspective to extract structures of an image. Moreover, compared to GM, PC is insensitive to the brightness and contrast. Here, we adopt the approach proposed by Kovesi [25] for PC computation. The specific computation of $\mathrm{PC}$ is given below.

Given a 1-D signal s, we denote $M_{n}^{e}$ and $M_{n}^{o}$ the even- and odd-symmetric filters on scales $n$, which form a quadrature pair. Responses of each quadrature pair to the signal will generate a response vector at position $j$ on scale $n:\left[e_{n}(j), o_{n}(j)\right]=\left[s(j) * M_{n}^{e}, s(j) * M_{n}^{o}\right]$, and the local amplitude on scale $n$ is $A_{n}(j)=\sqrt{e_{n}(j)^{2}+o_{n}(j)^{2}}$. Let $F(j)=\sum_{n} e_{n}(j)$ and $H(j)=\sum_{n} o_{n}(j)$, the $\mathrm{PC}$ can be computed as:

$$
P C(j)=\frac{U(j)}{\varepsilon+\sum_{n} A_{n}(j)}
$$

where $U(j)=\sqrt{F^{2}(j)+H^{2}(j)}$ and $\varepsilon$ is a small positive constant for avoiding division by zero. Usually, the spurious effect of noise should be excluded for PC computation, namely:

$$
P C(j)=\frac{(U(j)-T)^{+}}{\varepsilon+\sum_{n} A_{n}(j)}
$$

where $T$ represents the total noise influence that should be subtracted from $U(j),(\cdot)^{+}$denotes that the difference between the functions is not permitted to become negative. With the definition of 1-D PC, the 2-D PC at position $\mathbf{j}$ can 
be derived by integrating the 1-D PC from all orientations as:

$$
P C_{2 D}(\mathbf{j})=\frac{\sum_{o}\left(U_{o}(\mathbf{j})-T_{o}\right)^{+}}{\varepsilon+\sum_{o} \sum_{n} A_{n o}(\mathbf{j})}
$$

where $o$ denotes the index over orientations. At last, a sigmoid weighting function is introduced into Eq. 6 to adjust the $\mathrm{PC}$ value in each orientation as:

$$
P C_{2 D}(\mathbf{j})=\frac{\sum_{o}\left(W_{o}(\mathbf{j})\left(U_{o}(\mathbf{j})-T_{o}\right)^{+}\right)}{\varepsilon+\sum_{o} \sum_{n} A_{n o}(\mathbf{j})}
$$

where the weighting function $W(\mathbf{j})$ is defined as:

$$
W(\mathbf{j})=\frac{1}{1+e^{g(c-s(\mathbf{j}))}}
$$

where $c$ is the 'cut-off' value of the filter response spread, below which PC values will be penalized, $g$ is a gain factor which controls the sharpness of the cut-off. The spread function of $s(\mathbf{j})$ is given by:

$$
s(\mathbf{j})=\frac{1}{N} \frac{\sum_{n} A_{n}(\mathbf{j})}{\varepsilon+A_{\max }(\mathbf{j})}
$$

with $N$ being the considered total number of scales, $A_{\max }(\mathbf{j})$ the amplitude of the filter pair which have maximum response at $\mathbf{j}$. Interested readers can refer to 25] for more information of PC computation. Likewise, In Fig. 1, we show an example of the PC images extracted from the original and blurred images respectively. Among them, (h) is an original image without blur, (i) and (j) are the corresponding images with slight blur and heavy blur respectively. $(\mathrm{k})(\mathrm{l})(\mathrm{m})$ are the extracted PC images of $(\mathrm{h})(\mathrm{i})(\mathrm{j})$. The $\mathrm{PC}$ value distributions of these three images are illustrated in (n). It is observed from the PC images that PC can extract the structures in the images, and PC value degrades as the blur degree increases, which proves $\mathrm{PC}$ of the image can be used to indicate the blur level. Similarly, the PC value distribution of the heavy blur image is more divergent from that of the original image compared to the $\mathrm{PC}$ value distribution of the slight blur image, as shown in (n).

\subsection{Image quality estimation}

With the extracted GM and PC of the out-of-focus blurred image, we combine the GM and PC maps to get a localized structure map $S$ as:

$$
S(i, j)=\max \left\{G M(i, j) / G M_{\max }, P C(i, j)\right\}
$$




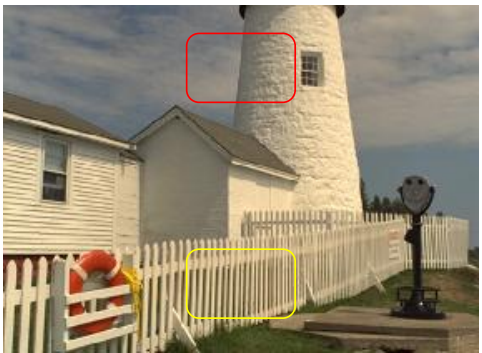

(a)

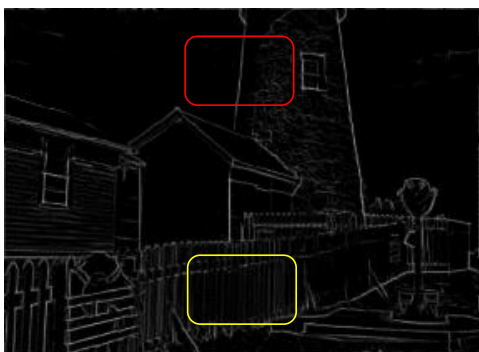

(c)

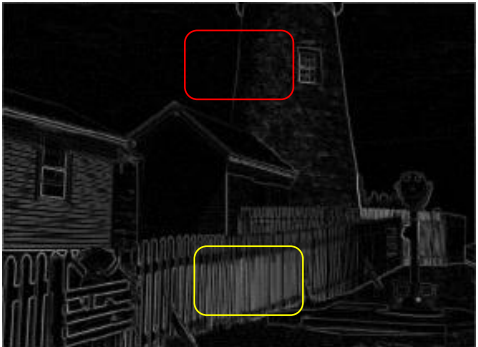

(b)

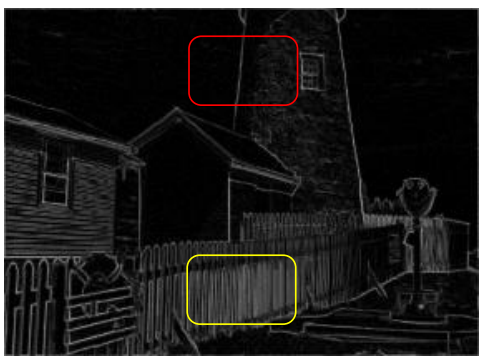

(d)

Figure 2: Illustration of the GM, PC and local structure S maps. (a) an example image, (b) GM map, (c) PC map, (d) S map.

where $(i, j)$ represents each position in $S, G M$ and $P C$ maps. As PC values are within $0-1$, we also normalize GM values to $0-1$ by dividing the GM values by $G M_{\max }$ which denotes the maximum value of $G M$, here $G M_{\max }=255 \sqrt{2}$. Then we take the maximum value of GM and PC values to form $S$ in that if anyone of GM and PC takes a larger value in each position, we think this position in the image is an structure feature point. Therefore, maximum combination of GM and PC guarantees that we can extract the structures in the image comprehensively. To illustrate this visually, we calculate GM and PC of the same image. The results are shown in Fig. 2. As can be observed, in the red rectangle area of (b), the edge of the tower cannot be extracted clearly. This is because both sides of the edge have similar luminance, namely in (a) the white cloud and the white tower are hard to distinguish in luminance. Therefore, GM can't characterize this edge well. While PC is able to capture this edge from the spectral domain. Similarly, in the yellow rectangle area of (c), the fences 175 have similar frequency behaviors, which make it difficult for PC to extract the 
structures. On the contrary, the structures of the fences can be revealed by GM. If we take the maximum value of GM and PC, we can obtain both the structures in the red rectangle and yellow rectangle in the meantime, verified in (d). Therefore, GM and PC can play complementary roles in structure extraction so that maximum combination of GM and PC enables us to extract the structures comprehensively. In the next, as stated before, the out-of-focus blur may exist irregularly over the image, which leads to inaccurate results of blurriness assessment, and thus the strategy we take here is to apply saliency detection to the out-of-focus blurred image towards emphasizing the regions that attract more visual attention for quality assessment. Specifically, we generate a corresponding saliency map of the out-of-focus blurred image through saliency detection, denoted by $S M$. The larger values in $S M$ means the co-located pixels in the out-of-focus blurred image are more significant to the HVS. Then we weight the localized structure map $S$ with $S M$ resulting in a localized quality map $Q$ :

$$
Q(i, j)=\frac{S M(i, j) \cdot S(i, j)}{\sum_{(i, j) \in Q} S M(i, j)}
$$

At last, we derive the quality score to evaluate the visual quality of out-of-focus blurred images based on percentile pooling strategy, which is widely adopted in IQA approaches [27, 20] [19]. Specifically, we take the root mean square of the $l \%$ largest values in the localized quality map $Q$ to define the GPSQ index as follows:

$$
G P S Q=\sqrt{\frac{1}{N} \sum_{(i, j) \in \Omega} Q^{2}(i, j)}
$$

where GPSQ gives the quality level of the out-of-focus blurred image, $\Omega$ contains all the positions of the $l \%$ largest values in $Q$ and $N$ counts the number of positions in $\Omega$. In our implementation, the saliency model Covsal [28] is employed for saliency prediction and $l$ is set to 20 as we suppose the least proportion for perceiving quality is $1 / 5$ [29]. It is noted that GPSQ belongs to NR methods. 


\subsection{Extension to color IQA}

Till now, the GPSQ index is designed on the luminance channel of the outof-focus blurred image. Since the chrominance information can also affect the HVS in quality evaluation of the distorted images. Therefore, we define GPSQ $c$

can't bring performance improvement while cause much more computational time. Then the local quality map is generated as:

$$
Q_{c}(i, j)=\frac{S M(i, j) \cdot S_{c}(i, j)}{\sum_{(i, j) \in Q_{c}} S M(i, j)}
$$

Finally, the $\mathrm{GPSQ}_{c}$ index is defined as:

$$
G P S Q_{c}=\sqrt{\frac{1}{N} \sum_{(i, j) \in \Omega} Q_{c}^{2}(i, j)}
$$

It is noted that the parameters for $\mathrm{GPSQ}_{c}$ are the same as that of GPSQ.

For intuitive understanding of GPSQ ${ }_{c}$, we show its flowchart in Fig. 3 clearly. 


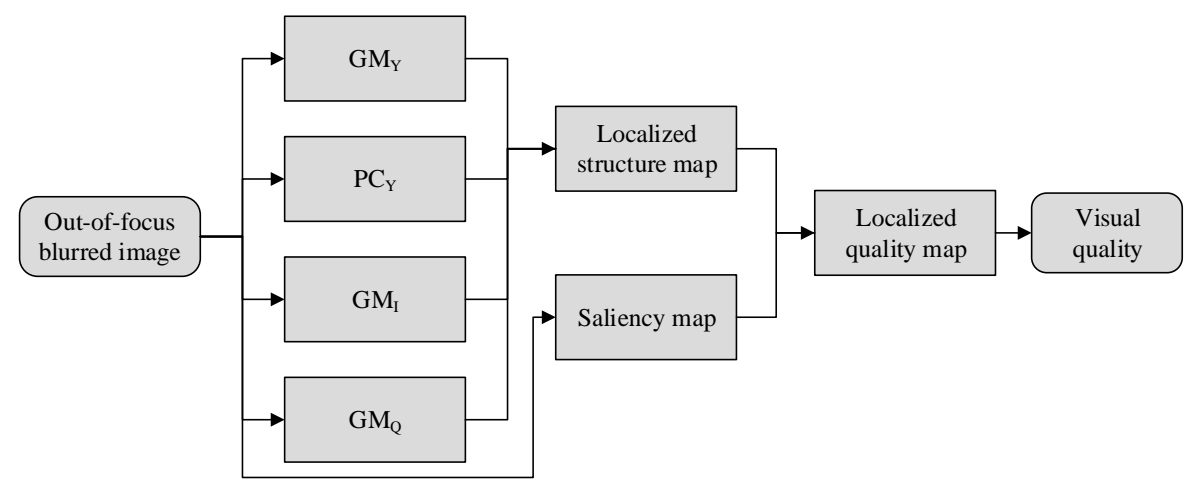

Figure 3: The flowchart of the proposed GPSQc.

As can be observed, given an out-of-focus blurred image, we calculate $\mathrm{GM}_{Y}$, $\mathrm{PC}_{Y}, \mathrm{GM}_{I}$ and $\mathrm{GM}_{Q}$ of the input image respectively. Then these four feature maps are combined to construct a localized structure map. Meanwhile, we perform saliency detection on the input out-of-focus blurred image and get a corresponding saliency map which locally indicates visual importance of the image. With the localized structure map and saliency map, we weight the structure map with the saliency map which leads to a localized quality map. At last, the localized quality map is pooled to produce the quality of the whole out-of-focus blurred image.

\section{Experiments}

This section will validate the effectiveness of the proposed method based on extensive experiments. Firstly, we introduce the experimental protocol and the image databases serving as the test bed for objective methods. Then we report the prediction results of the objective methods and deliver necessary analysis.

Furthermore, some important issues about GPSQ will also be discussed.

\subsection{Experimental protocol}

To estimate the prediction performance of the objective methods, we employ four commonly-adopted statistical indexes which are Kendall's rank correlation 
coefficient (KROCC), Spearman Rank order Correlation coefficient (SROCC),

root mean square error (RMSE) and Pearson's linear correlation coefficient (PLCC) respectively. These four indexes are all calculated between the subjective ratings and the objective scores given by the objective methods. The KROCC and SROCC values indicate the prediction monotonicity of the quality metric, PLCC reflects the prediction accuracy and RMSE points out the prediction consistency. Therefore, these four indexes demonstrate the prediction performance from different aspects. A superior IQA metric is expected to achieve values close to 1 in KROCC, SROCC and PLCC, while close to 0 in RMSE. As suggested by VQEG [30], before computing PLCC and RMSE, the objective scores are needed to be mapped to subjective ratings through nonlinear regression. Toward this end, we apply a logistic function with five parameters as:

$$
q(z)=\beta_{1}\left(\frac{1}{2}-\frac{1}{1+\exp \left(\beta_{2} \cdot\left(z-\beta_{3}\right)\right)}\right)+\beta_{4} \cdot z+\beta_{5}
$$

with $z$ and $q(z)$ being the input objective score and the mapped score. $\beta_{1}, \beta_{2} . . \beta_{5}$ are free parameters to be determined via the curve fitting process. Then these four indexes are respectively calculated as (Supposing $N$ denotes the total number of the testing images):

$$
S R O C C=1-\frac{6}{N\left(N^{2}-1\right)} \sum_{i=1}^{N} d_{i}^{2}
$$

where $d_{i}$ represents the distance between the $i$-th image's ranks in objective and subjective scores.

$$
K R O C C=\frac{2\left(N_{c}-N_{d}\right)}{N(N-1)}
$$

where $N_{c}$ and $N_{d}$ denote the number of concordant and discordant pairs in the testing database.

$$
P L C C=\frac{\sum_{i=1}^{N}\left(q_{i}-\bar{q}\right) \cdot\left(s_{i}-\bar{s}\right)}{\sqrt{\sum_{i=1}^{N}\left(q_{i}-\bar{q}\right)^{2} \cdot \sum_{i=1}^{N}\left(s_{i}-\bar{s}\right)^{2}}}
$$

where $s_{i}$ and $\bar{s}$ are the $i$-th image's subjective rating and the mean of the overall $s_{i}$. $q_{i}$ and $\bar{q}$ are the $i$-th image's mapped objective score through nonlinear 


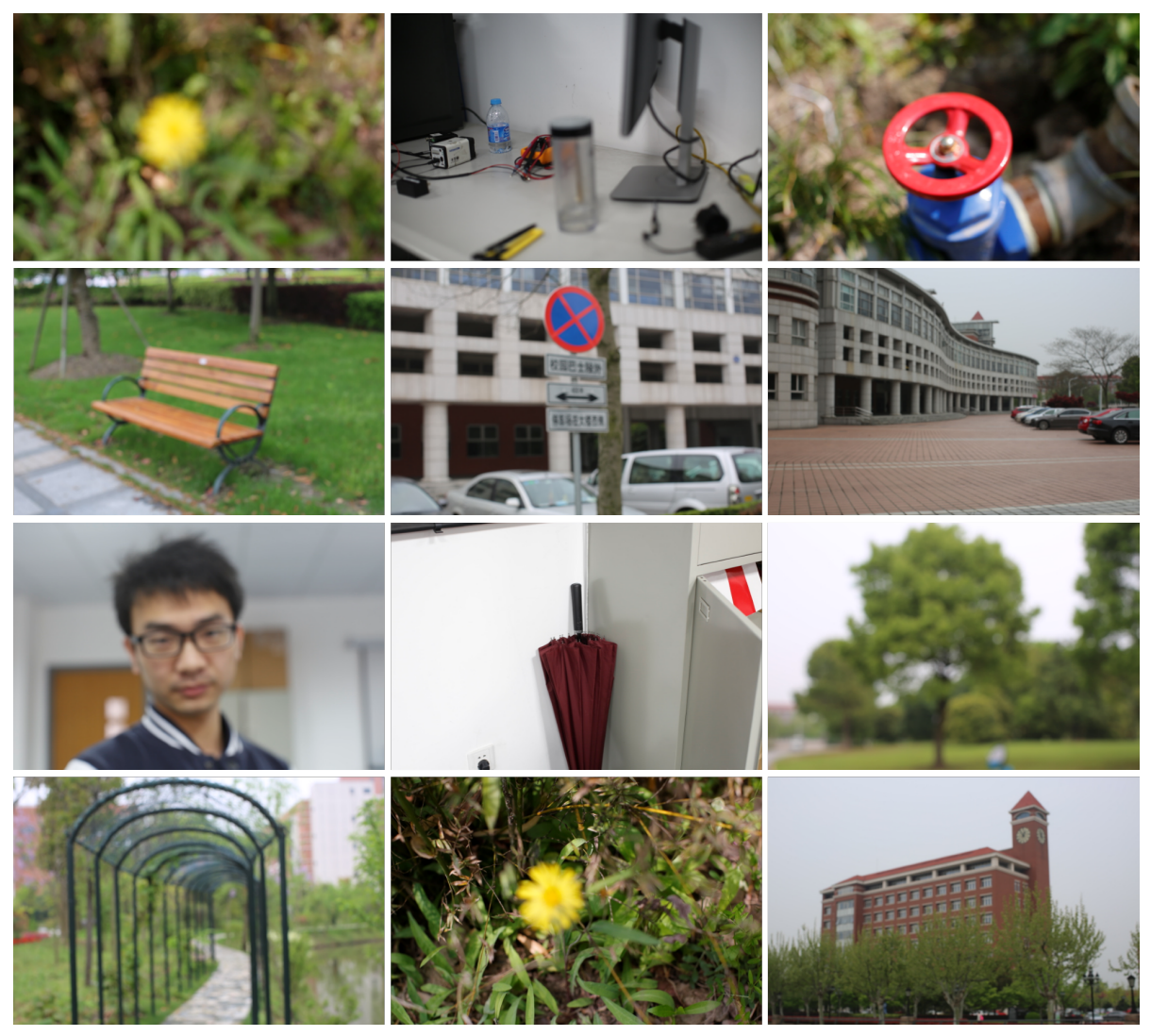

Figure 4: Example out-of-focus blurred images in out-of-focus blurred image dataset.

regression and the mean value. The last performance index RMSE is defined as:

$$
R M S E=\sqrt{\frac{1}{N} \sum_{i=1}^{N}\left(s_{i}-q_{i}\right)^{2}}
$$

where $s_{i}$ and $q_{i}$ represent the subjective rating and mapped objective score 265 respectively.

\subsection{Testing image dataset}

To the best of our knowledge, there are two real out-of-focus blurred image databases which can be employed to test our method. The first one is the out-of-focus blurred image dataset of our earlier work in [31. This dataset is 


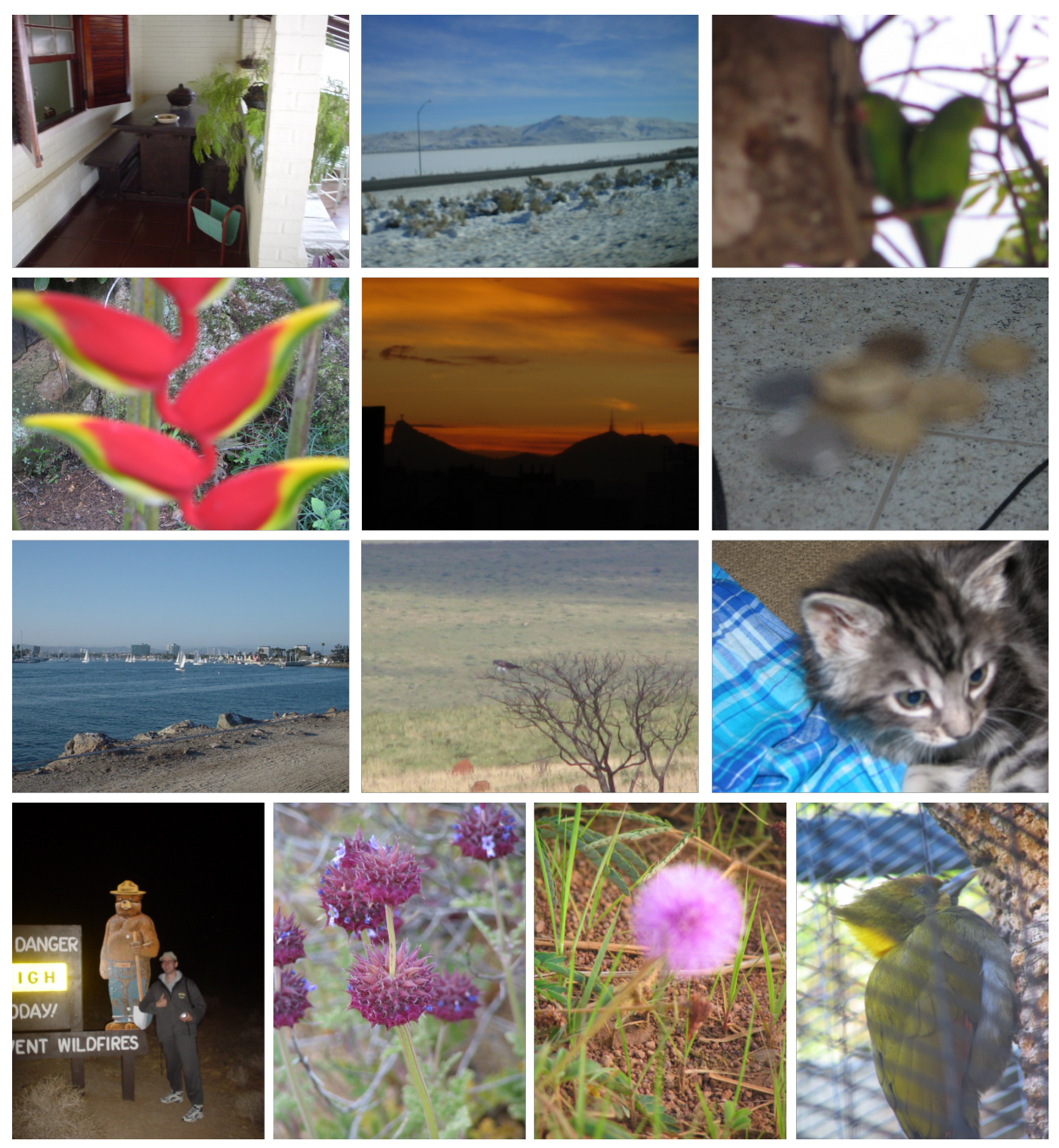

Figure 5: Example out-of-focus blurred images in BID database. 

were acquired by a single-lens camera. Then subjective test with Single-stimulus (SS) method was performed on this image dataset to collect subjective ratings for the images. Detailed information about this image dataset can be referred to 31 .

275 contains 585 images which can be classified into five blur cases, which are Unblurred, Out-of-focus, Simple Motion, Complex Motion and Other respectively. In our test, we chose the Out-of-focus class and the Other class which include the global and localized out-of-focus blurred images. In Fig. 4 , Fig. 5, we show some example out-of-focus blurred images in our dataset and BID database respectively. It can be observed that the real out-of-focus blur in the image is complex and spatially variant.

\subsection{Performance comparison}

As reported in Table 1 and Table 2, the prediction performance in terms of [33, BRISQUE [11, DESIQUE [34, DIIVINE [9], NFERM [12, NIQE [35, SISBLIM 36], BQMS [37, LPSI [38, CPBD 39], ARISMC [27], FISH [19], JNB [40], LPC [41] and S3 [20]. For clear comparison, we classify the competing methods into two types. The first type belongs to general-purpose NR IQA methods, which includes BIQI, BRISQUE, DESIQUE, DIIVINE, NFERM, NIQE, SISBLIM, BQMS and LPSI. The second type contains the representative methods specific to image blurriness assessment, which are CPBD, ARISMC, FISH, JNB, LPC and S3. As can be seen in Table 11, the first type generalpurpose NR methods can assess the quality of the out-of-focus blurred images 
Table 1: Overall prediction performance on our out-of-focus blurred image dataset

\begin{tabular}{ccccc}
\hline Methods & SROCC & KROCC & PLCC & RMSE \\
\hline BIQI [33] & 0.2580 & 0.1740 & 0.2241 & 1.2830 \\
BRISQUE [1] & 0.7361 & 0.5367 & 0.7263 & 0.9049 \\
DESIQUE [34] & 0.6899 & 0.4919 & 0.6811 & 0.9639 \\
DIIVINE [9] & 0.5865 & 0.4149 & 0.6475 & 1.0033 \\
NFERM [12] & 0.7756 & 0.5771 & 0.7529 & 0.8665 \\
NIQE [35] & 0.0906 & 0.0777 & 0.4693 & 1.1625 \\
SISBLIM [36] & 0.8554 & 0.6734 & 0.8155 & 0.7620 \\
BQMS [37] & 0.0455 & 0.0195 & 0.4161 & 1.1971 \\
LPSI [38] & 0.6590 & 0.4781 & 0.6957 & 0.9457 \\
\hline CPBD [39] & 0.7880 & 0.6009 & 0.7902 & 0.8068 \\
ARISMC [27] & 0.5356 & 0.3754 & 0.6932 & 0.9489 \\
FISH [19] & 0.8545 & 0.6665 & 0.8257 & 0.7426 \\
JNB [40] & 0.7369 & 0.5506 & 0.7306 & 0.8989 \\
LPC [41] & 0.8568 & 0.6620 & 0.8447 & 0.7047 \\
S3 [20] & 0.8641 & 0.6702 & 0.8431 & 0.7079 \\
GPSQ (Pro.) & $\mathbf{0 . 9 2 3 8}$ & 0.7515 & 0.9341 & 0.4700 \\
GPSQ (Pro.) & 0.9237 & $\mathbf{0 . 7 5 2 2}$ & $\mathbf{0 . 9 3 4 4}$ & $\mathbf{0 . 4 6 8 9}$ \\
\hline
\end{tabular}

300

\section{metlons} methods and outperforms them remarkably. In addition, $\mathrm{GPSQ}_{c}$ delivers better performance than GPSQ, which verifies the predicting quality can be further enhanced by taking color information into consideration.

Take a look at Table 2 we notice that the prediction performance decreases a lot for most of the objective methods. This can be mainly attributed to three points. First, the resolution of the images in BID out-of-focus blur database 


\begin{tabular}{ccccc} 
Table 2: Overall prediction performance on BID out-of-focus blurred image database \\
\hline Methods & SROCC & KROCC & PLCC & RMSE \\
\hline BIQI [33] & 0.4470 & 0.3077 & 0.5308 & 0.9100 \\
BRISQUE [11] & 0.0592 & 0.0417 & 0.3178 & 1.0181 \\
DESIQUE [34] & 0.1019 & 0.0720 & 0.1235 & 1.0656 \\
DIIVINE [9] & 0.2406 & 0.1647 & 0.2531 & 1.0388 \\
NFERM [12] & 0.2784 & 0.1846 & 0.3336 & 1.0123 \\
NIQE [35] & 0.3239 & 0.2147 & 0.3760 & 0.9950 \\
SISBLIM [36] & 0.0552 & 0.0324 & 0.3440 & 1.0083 \\
BQMS [37] & 0.1034 & 0.0667 & 0.1053 & 1.0678 \\
LPSI [38] & 0.2229 & 0.1495 & 0.3111 & 1.0205 \\
\hline CPBD [39] & 0.1640 & 0.1114 & 0.2712 & 1.0335 \\
ARISMC [27] & 0.1446 & 0.0953 & 0.1803 & 1.0562 \\
FISH [19] & 0.3097 & 0.2110 & 0.3282 & 1.0143 \\
JNB [40] & 0.0945 & 0.0639 & 0.2585 & 1.0373 \\
LPC [41] & 0.4280 & 0.2946 & 0.5147 & 0.9206 \\
S3 [20] & 0.3795 & 0.2575 & 0.4221 & 0.9734 \\
GPSQ (Pro.) & 0.4978 & 0.3434 & 0.5564 & 0.8922 \\
GPSQc (Pro.) & $\mathbf{0 . 4 9 7 9}$ & $\mathbf{0 . 3 4 3 6}$ & $\mathbf{0 . 5 5 7 1}$ & $\mathbf{0 . 8 9 1 8}$ \\
\hline
\end{tabular}

is varied, which can be viewed in Fig. 5 where the resolution of images in the last row is different from that of the images in the first three rows. While the resolution of the image indeed has an effect on its visual quality [42. The second point lies in that there possibly exists other kinds of distortion except out-of-focus blur in the image, such as motion blur. This will also affect the prediction accuracy of the objective methods. The last point owes to the fact that other factors can still influence subjective ratings for the visual quality. For instance, the image in the middle of the second row in Fig. 5 has low luminance, which disturbs subjective judgements of the image visual quality. In a word, there are other factors except out-of-focus blur which can also affect the quality of the image. While in the construction of our out-of-focus blurred image dataset, we eliminated the influence of other factors on the image quality as far as possible. To illustrate, we employed a tripod when acquiring the images 
Table 3: Prediction performance comparison of GM, PC and their combination

\begin{tabular}{ccccc}
\hline SE & SROCC & KROCC & PLCC & RMSE \\
\hline GM & 0.8996 & 0.7227 & 0.9126 & 0.5382 \\
PC & 0.8962 & 0.7184 & 0.9090 & 0.5488 \\
Combination & $\mathbf{0 . 9 2 3 8}$ & $\mathbf{0 . 7 5 1 5}$ & $\mathbf{0 . 9 3 4 1}$ & $\mathbf{0 . 4 7 0 0}$ \\
\hline
\end{tabular}

which can eliminate camera-shaken induced blur greatly. Besides, the resolution of images in our dataset is also fixed. Although the prediction performance of the methods decreases on the BID out-of-focus blurred database, our proposed GPSQ/GPSQ $c$ still performs the best among all the methods.

\subsection{Examination of $G M$ and $P C$ in $G P S Q$}

In Section 2.3. we analyzed GM and PC can play complementary roles in structure extraction and the maximum combination of them makes us fully extract the structures. Hence, combination of GM and PC is expected to better characterize the image blurriness so that the final quality prediction performance should be better than anyone of GM and PC. To verify this, we inspect the prediction performance of structure extraction with GM, PC and their combination respectively. Specifically, in GPSQ, we calculated GM, PC and their combination and examined the corresponding prediction performance on our out-of-focus blurred image dataset. In this process, other operations in GPSQ are all fixed. The experimental results are tabulated in Table 3 in which "SE" refers to structure extraction, the best performance is emphasized in boldface. From this table, we notice that the prediction accuracy of GM and PC is comparative as the values of SROCC, KROCC, PLCC and RMSE are very close. This indicates only calculating GM or PC for structure extraction leads to comparative prediction performance. While the performance of the combination of GM and PC is obviously better than anyone of them, which verifies the combination of GM and PC can noticeably improve the prediction performance. As GM and PC can extract the image structures complementarily, the maximum combination of them can fully extract the structures, which accounts for the prediction performance is superior to anyone of them. 
Table 4: SROCC values of different saliency models

\begin{tabular}{ccccc}
\hline Saliency & FISH & LPC & S3 & GPSQ \\
\hline None & 0.8545 & 0.8568 & 0.8641 & 0.8725 \\
SWD 43] & $\mathbf{0 . 9 0 3 0}$ & 0.8739 & $\mathbf{0 . 8 9 4 0}$ & 0.9091 \\
Covsal [28] & 0.8994 & 0.8710 & 0.8876 & $\mathbf{0 . 9 2 3 8}$ \\
GBVS [44] & 0.8842 & 0.8811 & 0.8773 & 0.9075 \\
Itti 45] & 0.8899 & 0.8811 & 0.8765 & 0.9054 \\
RCSS [46] & 0.8740 & $\mathbf{0 . 9 0 0 9}$ & 0.8654 & 0.9059 \\
RARE 47] & 0.8465 & 0.8470 & 0.8012 & 0.8590 \\
FES 48] & 0.8060 & 0.8151 & 0.7491 & 0.8195 \\
IS [49] & 0.8601 & 0.7483 & 0.8149 & 0.7764 \\
\hline
\end{tabular}

\subsection{The impact of saliency detection on prediction performance}

As we stated before, the complexity and irregularity of real out-of-focus blur give rise to difficulties for accurate evaluating the visual quality of the out-offocus blurred images. In this regard, we take the strategy of saliency prediction on the blurred images and emphasize the visually important regions for quality evaluation accordingly. Therefore, we want to testify the effectiveness of saliency prediction in quality estimation. In this experiment, without loss of generality, we tested 8 mainstream saliency models for saliency detection, which are SWD [43, Covsal [28, GBVS [44, Itti 45], RCSS [46, RARE [47, FES 48, and IS 49. In addition, we also included blurriness assessment methods for comparison, which are FISH, LPC and S3 as these three methods also produce a localized sharpness map of the blurred image that we can weight with saliency map like GPSQ. The manner of weighting the localized sharpness map with saliency map is kept the same as GPSQ in Eq. (11). The pooling operation to obtain the final quality is also fixed as described in Eq. (12). All the experiments were performed on our out-of-focus blurred image dataset and we list the experimental results in Table 4 . The prediction performance is evaluated in SROCC and the best one for each sharpness method in each column is stressed with boldface. "None" means we do not perform saliency detection and the quality is obtained by directly pooling the localized sharpness map. From the 


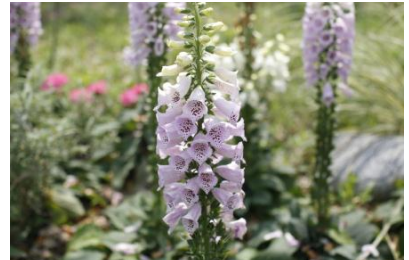

(a)

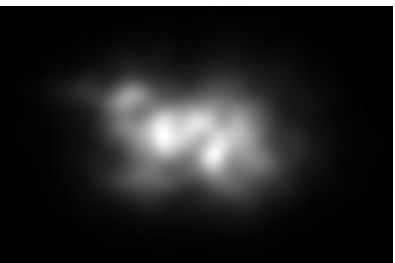

(b)

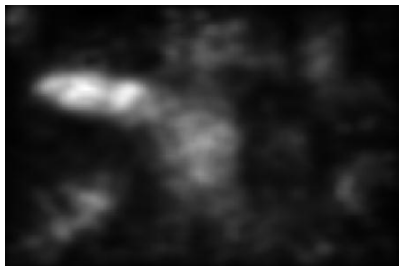

(c)

Figure 6: Example of saliency detection results. (a) an example image. (b) saliency map generated by Covsal. (c) saliency map generated by RARE.

observation of Table 4, we can find that for each sharpness assessment method, the SROCC values of SWD, Covsal, GBVS, Itti and RCSS are consistently better than that of "None", which verifies saliency detection is an effective strategy to deal with the complexity and irregularity of the out-of-focus blur in quality evaluation. However, the performance of RARE, FES and IS is still below that of "None", which can be attributed to that the prediction accuracy of these saliency models is not good enough to the out-of-focus blurred images. To account for this, we show an example of saliency prediction results in Fig. 6 It is observed that the saliency area generated by RARE focuses on the flowers in the background, which will mislead the quality evaluation result. On the contrary, Covsal produces more accurate result and will benefit the quality evaluation. By comparing each row, we notice that our proposed method GPSQ achieves the best performance in most cases, which can be owed to the superiority of GM and $\mathrm{PC}$ for characterizing the blurriness of the out-of-focus blurred image. As SROCC of Covsal attains the maximum value, we employ Covsal as the default saliency model in GPSQ experimentally.

\subsection{Complexity analysis}

Time complexity is an important attribute for the objective IQA methods. Therefore, we examine the computational complexity and time cost of all the competing methods. The experiments were performed on a Thinkpad X220 notebook with a $2.5 \mathrm{GHz} \mathrm{CPU}$ and $4 \mathrm{G}$ RAM. The software platform is Matlab 
Table 5: Computational complexity and running time comparison

\begin{tabular}{ccc}
\hline Methods & Time (s) & Complexity \\
\hline BIQI 33] & 0.36 & $O(N)$ \\
BRISQUE [1] & 0.22 & $O\left(d^{2} N\right)$ \\
DESIQUE [34] & 0.47 & $O\left(d^{2} N\right)$ \\
DIIVINE [9] & 26.43 & $O\left(N\left(\log N+m^{2}+N+392 b\right)\right)$ \\
NFERM [12] & 76.55 & $O\left(d^{2} N \log N\right)$ \\
NIQE [35] & 0.46 & $O\left(d^{2} N\right)$ \\
SISBLIM 36] & 4.51 & $O\left(d^{2} N \log N / 16\right)$ \\
BQMS [37] & 77.68 & $O\left(d^{2} N \log N\right)$ \\
LPSI [38] & 0.07 & $O(C)$ \\
\hline CPBD [39] & 0.84 & $O(N)$ \\
ARISMC [27] & 35.35 & $O\left(d^{2} N \log N\right)$ \\
FISH [19] & 0.08 & $O(N)$ \\
JNB [40] & 0.79 & $O(N)$ \\
LPC [41] & 1.67 & $O\left(180 N / k^{2}\right)$ \\
S3 [20] & 39.17 & $O\left(180 N / k^{2}\right)$ \\
GPSQ (Pro.) & 16.49 & $O(N-9(W+H)+81)$ \\
GPSQ (Pro.) & 16.54 & \\
\hline
\end{tabular}

R2012a. All the objective methods were tested on an image of $480 \times 720$ from our out-of-focus blurred image dataset and their running time was recorded

respectively. The experimental results are summarized in Table 5 , where $\mathrm{N}$ is the number of pixels in the image, $\mathrm{C}$ represents constant, $\mathrm{d}$ is the window size, $\mathrm{W}$ and $\mathrm{H}$ represent the width and height of the image, $\mathrm{m}$ is the DNT neighborhood size, $\mathrm{b}$ is the number of $2 \mathrm{D}$ histogram bin and $\mathrm{k}$ is the patch size. It can be observed that NFERM and BQMS spend the most time. This is because the AR prediction module in them is very time consuming. Since LPSI and FISH work in constant computational complexity, they can evaluate the image quality fastest. The computational time of our proposed method GPSQ/GPSQ exceeds 15 seconds as saliency detection takes a lot of time. Therefore, we envision that the speed of GPSQ/GPSQ ${ }_{c}$ can be further improved by embedding more efficient saliency detection methods in the future. 


\section{Conclusion}

In this paper, we have paid our attention to the visual quality assessment of images that suffer from out-of-focus blur. In detail, a dedicated quality assessment scheme, named GPSQ (Gradient magnitude and Phase congruencybased and Saliency-guided Quality model) was proposed to this end. In GPSQ, two low-level features from the spatial and spectral domain of the image, namely GM and PC were extracted and combined to characterize the image blurriness locally. Then saliency detection was performed on the out-of-focus blurred image to emphasize the visual important regions. At last, we derived a single quality score by pooling the localized quality map. We also extended GPSQ to GPSQ $c$ by incorporating the image chromatic information into GPSQ. Experimental results manifest that our proposed GPSQ/GPSQ ${ }_{c}$ acquires high correlation with subjective ratings for the quality of the out-of-focus blurred images.

Future works can be extended in two aspects: first, as deep learning reveal420 S outstanding ability in computer vision tasks, we can design more accurate methods via deep learning for precise quality assessment of the out-of-focus blurred images. There are two directions we can make our effort on. The one is to develop quality assessment methods via deep learning. The other one is to employ deep learning in developing saliency detection methods to accurately

425 predict what humans care about as we demonstrate accurate saliency maps can also benefit quality evaluation. Second, for a real image, there are still some other factors that will affect its visual quality except out-of-focus blur, such as jpeg compression, noise, contrast change etc. In this work, we have only studied on the factor of out-of-focus blur and the occasion of multiple factors is still not concerned. From the experiments we know that the prediction accuracy of our method on BID out-of-focus blurred image database is still far from ideal. Therefore, image quality evaluation under the influence of multiple factors becomes another problem worth studying. 


\section{References}

${ }_{435}$ [1] Z. Wang, A. C. Bovik, H. R. Sheikh, E. P. Simoncelli, Image quality assessment: from error visibility to structural similarity, IEEE Trans. Image Process. 13 (4) (2004) 600-612.

[2] L. Zhang, Y. Shen, H. Li, Vsi: A visual saliency-induced index for perceptual image quality assessment, IEEE Trans. Image Process. 23 (10) (2014) $4270-4281$.

[3] K. Gu, S. Wang, H. Yang, W. Lin, G. Zhai, X. Yang, W. Zhang, Saliencyguided quality assessment of screen content images, IEEE Trans. Multimedia 18 (6) (2016) 1098-1110.

[4] W. Zhang, A. Borji, Z. Wang, P. Le Callet, H. Liu, The application of visual saliency models in objective image quality assessment: A statistical evaluation, IEEE Trans. Neural Netw. Learn. Syst. 27 (6) (2016) 1266-1278.

[5] H. Alers, J. Redi, H. Liu, I. Heynderickx, Effects of task and image properties on visual-attention deployment in image-quality assessment, J. Electron. Imag. 24 (2) (2015) 023030-023030.

[6] R. Soundararajan, A. C. Bovik, Rred indices: Reduced reference entropic differencing for image quality assessment, IEEE Trans. Image Process. 21 (2) (2012) 517-526.

[7] G. Zhai, X. Wu, X. Yang, W. Lin, W. Zhang, A psychovisual quality metric in free-energy principle, IEEE Trans. Image Process. 21 (1) (2012) 41-52.

[8] X. Gao, W. Lu, D. Tao, X. Li, Image quality assessment based on multiscale geometric analysis, IEEE Trans. Image Process. 18 (7) (2009) 1409-1423.

[9] A. K. Moorthy, A. C. Bovik, Blind image quality assessment: From natural scene statistics to perceptual quality, IEEE Trans. Image Process. 20 (12) (2011) 3350-3364. 
[10] M. A. Saad, A. C. Bovik, C. Charrier, Blind image quality assessment: A natural scene statistics approach in the dct domain, IEEE Trans. Image Process. 21 (8) (2012) 3339-3352.

[11] A. Mittal, A. K. Moorthy, A. C. Bovik, No-reference image quality assessment in the spatial domain, IEEE Trans. Image Process. 21 (12) (2012) $4695-4708$

[12] K. Gu, G. Zhai, X. Yang, W. Zhang, Using free energy principle for blind image quality assessment, IEEE Trans. Multimedia 17 (1) (2015) 50-63.

[13] Z. Wang, H. R. Sheikh, A. C. Bovik, No-reference perceptual quality assessment of jpeg compressed images, in: Proc. IEEE Int. Conf. Image Process., 2002, pp. 477-480.

[14] L. Li, Y. Zhou, J. Wu, W. Lin, H. Li, Gridsar: Grid strength and regularity for robust evaluation of blocking artifacts in jpeg images, J. Vis. Commun. Image Represent. 30 (2015) 153-163.

[15] P. Marziliano, F. Dufaux, S. Winkler, T. Ebrahimi, A no-reference perceptual blur metric, in: Proc. IEEE Int. Conf. Image Process., 2002, pp. $57-60$.

[16] D. Zoran, Y. Weiss, Scale invariance and noise in natural images, in: Proc. IEEE Int. Conf. Comput. Vis., 2009, pp. 2209-2216.

[17] K. Gu, G. Zhai, X. Yang, W. Zhang, C. W. Chen, Automatic contrast enhancement technology with saliency preservation, IEEE Trans. Circuits Syst. Video Technol. 25 (9) (2015) 1480-1494.

[18] K. Gu, G. Zhai, W. Lin, M. Liu, The analysis of image contrast: From quality assessment to automatic enhancement, IEEE Trans. Cybernetics 46 (1) (2016) 284-297.

485

[19] P. V. Vu, D. M. Chandler, A fast wavelet-based algorithm for global and local image sharpness estimation, IEEE Signal Process. Lett. 19 (7) (2012) $423-426$. 
[20] C. T. Vu, T. D. Phan, D. M. Chandler, S3: A spectral and spatial measure of local perceived sharpness in natural images, IEEE Trans. Image Process. 21 (3) (2012) 934-945.

[21] L. Zhang, L. Zhang, X. Mou, D. Zhang, Fsim: a feature similarity index for image quality assessment, IEEE Trans. Image Process. 20 (8) (2011) $2378-2386$.

[22] Q. Wu, H. Li, F. Meng, K. N. Ngan, S. Zhu, No reference image quality assessment metric via multi-domain structural information and piecewise regression, J. Vis. Commun. Image Represent. 32 (2015) 205-216.

[23] Q. Wu, H. Li, F. Meng, K. N. Ngan, B. Luo, C. Huang, B. Zeng, Blind image quality assessment based on multichannel feature fusion and label transfer, IEEE Trans. Circuits Syst. Video Technol. 26 (3) (2016) 425-440.

[24] M. C. Morrone, J. Ross, D. C. Burr, R. Owens, Mach bands are phase dependent, Nature 324 (6094) (1986) 250-253.

[25] P. Kovesi, Image features from phase congruency, Videre: J. Comp. Vis. Res. 1 (3) (1999) 1-26.

[26] L. Henriksson, A. Hyvärinen, S. Vanni, Representation of cross-frequency spatial phase relationships in human visual cortex, J. Neurosci. 29 (45) (2009) 14342-14351.

[27] K. Gu, G. Zhai, W. Lin, X. Yang, W. Zhang, No-reference image sharpness assessment in autoregressive parameter space, IEEE Trans. Image Process. 24 (10) (2015) 3218-3231.

[28] E. Erdem, A. Erdem, Visual saliency estimation by nonlinearly integrating features using region covariances, J. Vis. 13 (4) (2013) 11.

[29] K. Gu, W. Lin, G. Zhai, X. Yang, W. Zhang, C. W. Chen, No-reference quality metric of contrast-distorted images based on information maximization, IEEE Trans. Cybernetics PP (99) (2016) 1-7. 
${ }_{515}$ [30] A. M. Rohaly, J. Libert, P. Corriveau, A. Webster, et al., Final report from the video quality experts group on the validation of objective models of video quality assessment, ITU-T Standards Contribution COM (2000) $9-80$.

[31] Y. Liu, G. Zhai, X. Liu, D. Zhao, Quality assessment for out-of-focus blurred images, in: Visual Communications and Image Processing (VCIP), 2015, IEEE, 2015, pp. 1-4.

[32] A. Ciancio, A. L. N. T. da Costa, E. A. da Silva, A. Said, R. Samadani, P. Obrador, No-reference blur assessment of digital pictures based on multifeature classifiers, IEEE Trans. Image Process. 20 (1) (2011) 64-75.

525 [33] A. K. Moorthy, A. C. Bovik, A two-step framework for constructing blind image quality indices, IEEE Signal Process. Lett. 17 (5) (2010) 513-516.

[34] Y. Zhang, D. M. Chandler, No-reference image quality assessment based on log-derivative statistics of natural scenes, J. Electron. Imag. 22 (4) (2013) 043025-043025.

[35] A. Mittal, R. Soundararajan, A. C. Bovik, Making a completely blind image quality analyzer, IEEE Signal Process. Lett. 20 (3) (2013) 209-212.

[36] K. Gu, G. Zhai, X. Yang, W. Zhang, Hybrid no-reference quality metric for singly and multiply distorted images, IEEE Trans. Broadcast. 60 (3) (2014) 555-567.

${ }_{535}$ [37] K. Gu, G. Zhai, W. Lin, X. Yang, W. Zhang, Learning a blind quality evaluation engine of screen content images, Neurocomputing 196 (2016) 140-149.

[38] Q. Wu, Z. Wang, H. Li, A highly efficient method for blind image quality assessment, in: IEEE Int. Conf. Image Process., 2015, pp. 339-343.

${ }_{540}[39]$ N. D. Narvekar, L. J. Karam, A no-reference image blur metric based on the cumulative probability of blur detection (cpbd), IEEE Trans. Image Process. 20 (9) (2011) 2678-2683. 
[40] R. Ferzli, L. J. Karam, A no-reference objective image sharpness metric based on the notion of just noticeable blur (jnb), IEEE Trans. Image Process. 18 (4) (2009) 717-728.

[41] R. Hassen, Z. Wang, M. M. Salama, Image sharpness assessment based on local phase coherence, IEEE Trans. Image Process. 22 (7) (2013) 27982810.

[42] K. Gu, M. Liu, G. Zhai, X. Yang, W. Zhang, Quality assessment considering viewing distance and image resolution, IEEE Trans. Broadcast. 61 (3) (2015) 520-531.

[43] L. Duan, C. Wu, J. Miao, L. Qing, Y. Fu, Visual saliency detection by spatially weighted dissimilarity, in: Proc. IEEE Int. Conf. Comput. Vis. Pattern Recog., 2011, pp. 473-480.

555 [44] J. Harel, C. Koch, P. Perona, Graph-based visual saliency, in: Proc. Adv. Neural Inform. Process. Syst., 2006, pp. 545-552.

[45] L. Itti, C. Koch, E. Niebur, A model of saliency-based visual attention for rapid scene analysis, IEEE Trans. Pattern Anal. Mach. Intell. 20 (11) (1998) 1254-1259.

[46] T. N. Vikram, M. Tscherepanow, B. Wrede, A saliency map based on sampling an image into random rectangular regions of interest, Pattern Recognit. 45 (9) (2012) 3114-3124.

[47] N. Riche, M. Mancas, B. Gosselin, T. Dutoit, Rare: A new bottom-up saliency model, in: IEEE Int. Conf. Image Process., 2012, pp. 641-644.

[48] K. Gu, G. Zhai, W. Lin, X. Yang, W. Zhang, Visual saliency detection with free energy theory, IEEE Signal Process. Lett. 22 (10) (2015) 1552-1555.

[49] X. Hou, J. Harel, C. Koch, Image signature: Highlighting sparse salient regions, IEEE Trans. Pattern Anal. Mach. Intell. 34 (1) (2012) 194-201. 